\title{
Prevalence of Tuberculosis among HIV Positive Individuals with Asymptomatic Disease States at St. Paul's Hospital Millennium Medical College, Addis Ababa, Ethiopia
}

Semaria Solomon ${ }^{1 *}$, Yared Asmare', Bekele Taddesse ${ }^{1}$, Shewalem Negah', Yeshiwendem Mamuye ${ }^{1}$, Berehanu Yitayew ${ }^{3}, Z^{1}{ }^{1}$ alem Yaregal ${ }^{2}$, Ephrem Tesfaye ${ }^{2}$ and Abebaw Kebede ${ }^{2}$

${ }^{1}$ St. Paul's Hospital Millennium Medical College, Ethiopia

${ }^{2}$ Ethiopian Public Health Institute, Ethiopia

${ }^{3}$ Debre Brehan University, Ethiopia

\begin{abstract}
Background: There is evidence that symptom screening for TB in HIV positive individuals misses the appropriate investigative procedures to confirm for proper diagnosing of TB. High value should be placed in ensuring that TB is diagnosed early in HIV positive individuals, which have an increased likelihood of having undetected TB and high risk of poor health outcomes in the absence of early diagnosis and treatment.

Objective: The aim of the study was to determine the prevalence of TB among HIV positive individuals with asymptomatic states at St. Paul's Hospital millennium Medical College Addis Ababa, Ethiopia.

Methods: A cross-sectional study was conducted from May to August, 2014 at SPHMMC. The study included 95 (34 male and 61 female) HIV positive individuals with no TB symptoms (current cough, fever, night sweat, and weight loss). Structured questionnaire was used to collect socio-demographic and clinical related data. The prevalence of TB was determined on the basis of AFB, Xpert MTB/RIF findings and diagnosis with chest X-ray.

Result: Out of the 95 participants, the prevalence of undiagnosed Tuberculosis among asymptomatic HIVpatients was $1.1 \%$. The socio-demographic characteristics and related risk factors were not significantly associated with TB finding rate by smear positive and Xpert assay. Our finding also showed a $27 \%$ abnormal chest X- ray suggestive of TB. In these HIV positive participants, presence of a patient with previous TB history was significantly abnormal by chest X-ray finding than a patient without TB history.

Conclusion: The present finding of asymptomatic undiagnosed Pulmonary TB among HIV-patient is 1.1\% (both with AFB and Xpert) in the study area. This showed there is a chance of transmissions of TB to contacts before the proper diagnosis and treatment is made. While the symptom screening algorithm missed the $1.1 \%$ TB cases, the AFB technique was able to detect the asymptomatic and missed TB case that was also detected by Xpert assay. TB control programs must consider TB disease prevalence when estimating the possible performance of any screening algorithm. National TB control program should weigh the risks, benefits and cost of screening all asymptomatic and symptomatic HIV infected individuals for TB by Xpert assay.
\end{abstract}

Keywords: Tuberculosis; HIV; Asymptomatic

Abbreviations: AFB: Acid-Fast Bacilli; AIDS: Acquired Immuno Deficiency Syndrome; ART: Antiretroviral Therapy; CDC: Center for Disease Control and Prevention; CT: Computed Tomography; CXR: $\quad$ Chest Radiography; DST: Drug Susceptibility Test; EPTB: Extra Pulmonary Tuberculosis; HAART: Highly Active Antiretroviral Therapy; HIV: Human Immunodeficiency Virus; IGRA: InterferonGamma Release Assay; INH: Isoniazid; IPT: Isoniazid Preventive Therapy; LJ: Lowenstein-Jensen; MDG: Millennium Development Goal; MDR: Multidrug Resistance; MoH: Ministry of Health; MTB: Mycobacterium tuberculosis; NAAT: Nucleic Acid Amplification Test; OIs: Opportunistic Infections; PCR: Polymerase Chain Reaction; PHCU: Primary Health Care Unit; PLWHA: People Living with HIV/ AIDS; PTB: Pulmonary Tuberculosis; RIF: Rifampicin; SPHMMC: St. Paul's Hospital Millennium Medical College; SPSS: Statistical Package for the Social Sciences; TB: Tuberculosis; TST: Tuberculin Skin Test interferon-gamma release assay (IGRA); WHO: World Health Organization; XDR: Extensively Drug Resistant

\section{Background}

Tuberculosis (TB) claims about two million lives per year, remains one of the leading causes of death among the infectious diseases and is the leading killer of people with AIDS [1]. HIV is one of the risk factors for developing TB disease in those with latent or new Mycobacterium tuberculosis (MTB) infection. It remains the world's most important cause of death from an infectious agent, besides the human immunodeficiency virus (HIV) with which it is intimately linked. The risk of developing TB is between 20 and 37 times greater in people living with HIV than among those who do not have HIV infection. TB is responsible for more than a quarter of deaths in people living with

*Corresponding author: Semaria Solomon, MSc in Medical Mirobiology, Principal Investigator, St.Paul's Hospital Millennium Medical College, Addis Ababa, Ethiopia, Tel: +251-911217830; E-mail: semariasol@gmail.com

Received May 10, 2015; Accepted May 26, 2015; Published May 28, 2015

Citation: Solomon S, Asmare Y, Taddesse B, Negah S, Mamuye Y, et al. (2015) NPrevalence of Tuberculosis among HIV Positive Individuals with Asymptomatic Disease States at St. Paul's Hospital Millennium Medical College, Addis Ababa, Ethiopia. J Med Microb Diagn 4: 192. doi:10.4172/21610703.1000192

Copyright: (c) 2015 Solomon S, et al. This is an open-access article distributed under the terms of the Creative Commons Attribution License, which permits unrestricted use, distribution, and reproduction in any medium, provided the original author and source are credited. 
Citation: Solomon S, Asmare Y, Taddesse B, Negah S, Mamuye Y, et al. (2015) Prevalence of Tuberculosis among HIV Positive Individuals with Asymptomatic Disease States at St. Paul's Hospital Millennium Medical College, Addis Ababa, Ethiopia. J Med Microb Diagn 4: 192. doi:10.4172/21610703.1000192

HIV [2]. TB control is high on the international public health agenda, not just because of the enormous burden of the disease, but also because the appropriate initiation of short-course chemotherapy is recognized to be among the most cost-effective of all health interventions $[3,4]$.

The Millennium Development Goal (MDG) 6, on combating HIV/ AIDS, malaria and TB, has three specific targets. Two targets on HIV/ AIDS aim at halting and beginning to reverse the spread of HIV/AIDS and to ensure universal access to treatment of HIV/AIDS for those who need treatment. The third target seeks the spread of malaria and TB by 2015 [5]. To halt and begin to reverse TB will require the successful development and deployment of at least some of the new tools described in the Global Plan. Improved TB diagnostics, to shorten diagnostic delays for patients with active TB and perhaps to halt the development of MDR/XDR-TB [6]. WHO algorithm suggests all people living with HIV in HIV prevalent and resource limited settings to be screened for TB symptoms (cough, fever, weight loss and night sweat). The presence of any one or combination of these symptoms should be investigated based on the national guideline (smear microscopy and culture in Ethiopian context). Chest radiography can be done if available, but is not required to classify patients into TB and non-TB groups. In high HIV-prevalence settings with a high TB prevalence among people living with HIV (e.g. greater than 10\%), strong consideration must be given to adding other sensitive investigations. In contrary, HIV positive individuals with no TB symptoms will be subjected to Isoniazid Preventive Therapy (IPT) following assessment for contraindications [2]. In 2011, WHO has recommended in its rapid implementation of the Xpert MTB/RIF diagnostic test guideline that "All persons living with HIV who have signs or symptoms of TB, those seriously ill and suspected of having TB regardless of HIV status, and those with unknown HIV status presenting with strong clinical evidence of HIV infection in HIV prevalent settings should receive an Xpert MTB/RIF test as a primary diagnostic test." The specific diagnosis of TB had also depended one way or another on the demonstration that $M$. tuberculosis or a fragment of the organism is present in a clinical specimen. Man resource-limited settings have developed a heavy dependence on the acid-fast bacillus (AFB) smear microscopy. Unfortunately several technical limitations of this test have led to the under diagnosis of active cases of TB when it is the only confirmatory test. In many settings, the limited sensitivity of the AFB smear is augmented by a variety of conventional but less specific methods of diagnosis, such as clinical evaluation, chest radiographs, and chest computed tomography (CT) scans. This practice often leads to over diagnosis of active TB [7]. Gold standard tests confirming disease require the detection of $M$. tuberculosis (MTB) in respiratory or other body samples, either in form of isolation by culture or detection of MTB- spe cific nucleic acids by molecular methods. When such methods were not available, diagnosis was typically based on microscopy to identify AFB in sputum smears, which is limited by a sensitivity of $50 \%$ or less and detects mostly advanced stages of pulmonary cavitary disease [8]. Recently, WHO has endorsed Xpert MTB/RIF test for the detection MTB and drug resistant TB. It is an integrated fully automated specimen processing and nucleic acid amplification test (NAAT). The assay is highly sensitive, specific and rapid with turnaround time of only two hours. The impact of this highly sensitive diagnostic tool was not studied well for the detection of TB among HIV positive individuals with atypical TB symptoms. High value should be placed in ensuring that TB is diagnosed early in HIV positive individuals, which have a high likelihood of having undetected TB and high risk of poor health outcomes in the absence of early diagnosis and treatment. Recognition of asymptomatic TB allows for the timely initiation of treatment that appears to reduce mortality

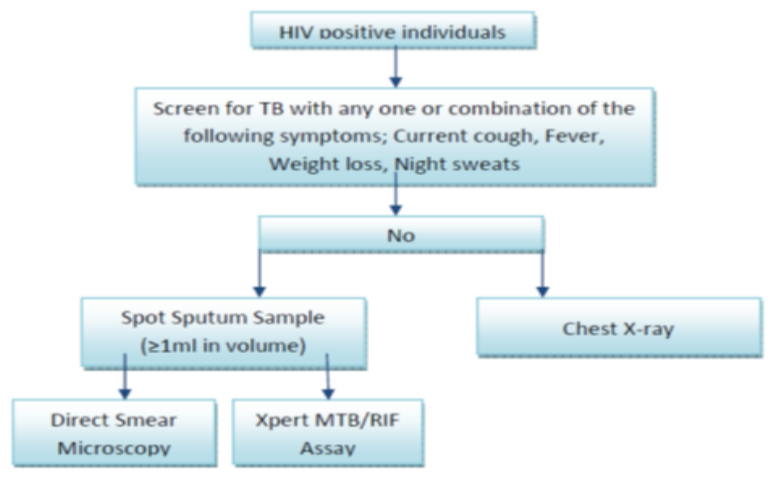

Figure 1: Flow chart for diagnosing HIV positive individuals with no symptoms of TB.

\begin{tabular}{|c|c|c|}
\hline Character & Frequency & Percent \\
\hline \multicolumn{3}{|c|}{ Age } \\
\hline$\leq 25$ & 4 & 4.2 \\
\hline $26-35$ & 43 & 45.3 \\
\hline $36-45$ & 25 & 26.3 \\
\hline $46-55$ & 18 & 18.9 \\
\hline$\geq 56$ & 5 & 5.3 \\
\hline \multicolumn{3}{|l|}{ Sex } \\
\hline Male & 34 & 35.8 \\
\hline Female & 61 & 64.2 \\
\hline \multicolumn{3}{|c|}{ Marital status } \\
\hline Single & 21 & 22.1 \\
\hline Married & 32 & 33.7 \\
\hline Separated & 8 & 8.4 \\
\hline Divorced & 15 & 15.8 \\
\hline Widowed & 19 & 20 \\
\hline \multicolumn{3}{|c|}{ Educational status } \\
\hline Illiterate & 19 & 20 \\
\hline Literate & 8 & 8.4 \\
\hline Elementary education & 24 & 25.3 \\
\hline Secondary education & 35 & 36.8 \\
\hline Higher education & 9 & 9.5 \\
\hline \multicolumn{3}{|c|}{ Occupation } \\
\hline Housewife & 22 & 23.2 \\
\hline Government employee & 12 & 12.6 \\
\hline Private employee & 37 & 38.9 \\
\hline Petty trader & 3 & 3.2 \\
\hline Unemployed & 21 & 22.1 \\
\hline \multicolumn{3}{|c|}{ Religion } \\
\hline Orthodox & 69 & 72.6 \\
\hline Muslim & 16 & 16.8 \\
\hline Protestant & 10 & 10.5 \\
\hline \multicolumn{3}{|c|}{ Ethnicity } \\
\hline Oromo & 30 & 31.6 \\
\hline Amhara & 41 & 43.2 \\
\hline Gurage & 14 & 14.7 \\
\hline Tigre & 7 & 7.4 \\
\hline
\end{tabular}

Table 1: Socio demographic characteristics of 95 study participants at SPHMMC, Addis Ababa Ethiopia, 2014

as there is a noted increased risk of death in the group. The proper diagnosis also prevents inappropriate administration of IPT where this mono-therapy could predispose the patient for the development of drug resistance TB and ineffective treatment outcome. In HIV positive population, asymptomatic $\mathrm{TB}$ appears to be rapidly progressive if 
Citation: Solomon S, Asmare Y, Taddesse B, Negah S, Mamuye Y, et al. (2015) Prevalence of Tuberculosis among HIV Positive Individuals with Asymptomatic Disease States at St. Paul's Hospital Millennium Medical College, Addis Ababa, Ethiopia. J Med Microb Diagn 4: 192. doi: $10.4172 / 21610703.1000192$

Page 3 of 6

\begin{tabular}{|c|c|c|}
\hline Characteristics & Frequency & Percent \\
\hline \multicolumn{3}{|c|}{ Drinking } \\
\hline Yes & 3 & 3.2 \\
\hline No & 92 & 96.8 \\
\hline \multicolumn{3}{|c|}{ Smoking } \\
\hline Yes & 1 & 1.1 \\
\hline No & 94 & 96.8 \\
\hline \multicolumn{3}{|c|}{ TB contact } \\
\hline Yes & 9 & 9.5 \\
\hline No & 86 & 90.5 \\
\hline \multicolumn{3}{|c|}{ With whom } \\
\hline Family & 5 & 5.3 \\
\hline Friends & 3 & 3.2 \\
\hline Prison & 6 & 6.3 \\
\hline Previous TB history & 44 & 46.3 \\
\hline \multicolumn{3}{|c|}{ Treated } \\
\hline Completed & 43 & 45.3 \\
\hline Defaulter & 1 & 1.1 \\
\hline \multicolumn{3}{|c|}{ HIV Stage } \\
\hline 1 & 85 & 89.5 \\
\hline II & 3 & 3.2 \\
\hline III & 6 & 6.3 \\
\hline IV & 1 & 1.1 \\
\hline \multicolumn{3}{|l|}{ ART } \\
\hline Yes & 93 & 96.8 \\
\hline No & 3 & 3.2 \\
\hline \multicolumn{3}{|c|}{ Current Regimen } \\
\hline $\begin{array}{c}\text { 1c AZT (Zidovudine), 3TC(Lamuvidine), } \\
\text { NVP (Nevrapine) }\end{array}$ & 13 & 13.9 \\
\hline $\begin{array}{c}\text { 1d AZT (Zidovudine), 3TC(Lamuvidine), } \\
\text { EFV(Efaverine) }\end{array}$ & 15 & 16.1 \\
\hline $\begin{array}{c}\text { 1e TDF (Tenofovir), 3TC (Lamuvidine), } \\
\text { EFV (Efaverine) }\end{array}$ & 42 & 45.2 \\
\hline $\begin{array}{c}\text { 1f TDF (Tenofovir), 3TC (Lamuvidine), } \\
\text { NVP (Nevrapine) }\end{array}$ & 1 & 1.1 \\
\hline \multicolumn{3}{|l|}{ CD4 } \\
\hline$\leq 350$ & 32 & 33.7 \\
\hline$>350$ & 63 & 66.3 \\
\hline
\end{tabular}

Table 2: Distributions of lifestyle and clinical history of 95 study participants at SPHMMC, Addis Ababa Ethiopia 2014.

left untreated. This study attempts to determine the prevalence of asymptomatic TB in HIV positive individuals with the recently WHO recommended rapid molecular assay, Xpert MTB/RIF, for a better detection MTB and rifampicin resistance. To this date, there is no study conducted on asymptomatic individuals with the above mentioned molecular technique [9-15].

\section{Methods}

A cross-sectional study was conducted from May to August, 2014 at St. Paul's Hospital Millennium Medical College, Addis Ababa, Ethiopia among adult HIV/AIDS patients attending the outpatient Department of ART clinic. The hospital has 309 beds for inpatients, rendering referral health services for over 1,500,000 inhabitants. It provides inpatient and outpatient services, including care and treatment for TB and HIV/AIDS patients. At the time of this study, there were 5,537 HIV positive adults on follow up in the ART clinic, of these 1,541 patients were on pre ART and 3,996 patients have started ART. Moreover, there were eight TB diagnosed patients on treatment in the ART clinic. Ninety five individuals who are HIV positives, $\geq 18$ years old and found to be asymptomatic were the study subjects. Ninety five consecutive patients, with a point estimate prevalence calculation, that were HIV positive but asymptomatic for TB were included. Data was collected using semi-structured questionnaires for socio- demographic and clinical data including TB and HIV treatment history, CD4 counts and radiology findings. The questionnaire was pre-tested in $5 \%$ of the study subjects which were not included in the study, and an appropriate modification was made based on the findings. Data was collected by trained data collectors. Eligible participants were requested to give one spot sputum sample for AFB and Xpert MTB/RIF analysis. Xpert MTB/ RIF assay was carried out at the National TB Reference Laboratory (NRL), Ethiopian Public Health Institute (EPHI) (Figure 1).

\section{Result}

A total of 96 participants were enrolled, out of these all of the participants responded to the questionnaire but one patient's sputum sample was lost, making the response rate $98.95 \%$. The mean age of the participants was $38.64(\mathrm{SD}, 9.4)$. The age range was from 21 to 70 years of age. The majority were female (64.2\%), married (33.7\%) and had secondary education (36.8\%). Thirty seven of the individuals were private employees, $(43.2 \%)$ were Amhara and (72.6\%) were orthodox (Table 1). Of the 95 participants, 3.2\% drank alcohol, of which $2.1 \%$ drank 1-2 per day, while $1.1 \%$ drank 2-4. Meanwhile, with regards to smoking, only one individual smoked 1 to 2 packs per day. Nine persons had previous TB contact of which $6.3 \%$ had contact in prison, $5.3 \%$ with family and $3.2 \%$ with friends. Almost half (46.3\%) had a previous history of $\mathrm{TB}$, of which only one was a defaulter, while all the others, had completed treatment. By and large, $89.5 \%$ of the enrolled were on HIV stage one. Participants who were Pre ART were only $3.2 \%$. The most prevalent regiment was $1 \mathrm{e}(\mathrm{TDF}+3 \mathrm{TC}+\mathrm{EFV})$ with 42 of the participants. The CD4 lymphocyte count was known for 95 of the participants with a minimum of 51 and $66.3 \%$ had a CD4 count of greater than 350 (Table 2). Only one patient was positive with both Ziehl Neelson (ZN) sputum AFB microscopy and Xpert MTB/RIF assay from the 95 participants screened. This makes the prevalence (1.1\%), while all the rest were negative by both methods. CD4 result was obtained for the 95 participants, $66.3 \%$ had a CD4 of greater than 350. There was no significant association of CD4 with TB occurrence and chest $\mathrm{X}$ ray (CXR) result.

From the 95 participants who were screened for CXR, 21 individuals had an abnormal CXR finding which is potentially compatible with

\begin{tabular}{|c|c|c|}
\hline Characteristics & Frequency & Percent \\
\hline Cavitary disease & 2 & 2.1 \\
\hline Milary TB & 1 & 1.1 \\
\hline Effusion & 6 & 6.3 \\
\hline Hilar lad & 3 & 3.2 \\
\hline Fibrosis & 10 & 10.5 \\
\hline Consolidation & 6 & 6.3 \\
\hline Infiltrate & 8 & 8.4 \\
\hline Nodule & 5 & 5.3 \\
\hline Calcified granuloma & 1 & 1.1 \\
\hline Non calcified granuloma & 0 & 0 \\
\hline Final chest X ray result & & \\
\hline Normal & 69 & 72.6 \\
\hline Abnormal with non pulmonary disease & 5 & 5.3 \\
\hline Abnormal with pulmonary disease & 21 & 21.1 \\
\hline Highly suggestive TB & 6 & 6.3 \\
\hline Probable TB & 11 & 11.5 \\
\hline Non TB lung disease & 4 & 4.2 \\
\hline
\end{tabular}

Table 3: Chest X-ray findings of 95 study participants at SPHMMC, Addis Ababa Ethiopia, 2014. 
Citation: Solomon S, Asmare Y, Taddesse B, Negah S, Mamuye Y, et al. (2015) Prevalence of Tuberculosis among HIV Positive Individuals with Asymptomatic Disease States at St. Paul's Hospital Millennium Medical College, Addis Ababa, Ethiopia. J Med Microb Diagn 4: 192. doi:10.4172/21610703.1000192

Page 4 of 6

\begin{tabular}{|c|c|c|c|c|c|c|c|}
\hline \multirow[t]{2}{*}{ Character } & \multirow[t]{2}{*}{$\mathrm{N}=95 / \%$} & \multirow{2}{*}{$\begin{array}{l}\text { Chest X-Ray Final } \\
\text { Result } \\
\text { Normal(n=69) } \\
\end{array}$} & & COR(95\%Cl) & P-Value & AOR(95\%Cl) & P-Value \\
\hline & & & \multicolumn{2}{|l|}{ Abnormal $(n=26)$} & & & \\
\hline \multicolumn{8}{|l|}{ Sex } \\
\hline Female & $61(64.2)$ & $45(73.8 \%)$ & $16(26.2 \%)$ & $1.2(0.46-2.9)$ & 0.74 & & \\
\hline Male & $34(35.8)$ & $24(70.5 \%)$ & $10(29.4 \%)$ & 1 & & & \\
\hline \multicolumn{8}{|l|}{ Age } \\
\hline$\leq 40$ & $63(66.3)$ & $49(77.7 \%)$ & $14(22.2 \%)$ & 1 & & & \\
\hline$\geq 41$ & $32(33.7)$ & $20(62.5 \%)$ & $12(37.5 \%)$ & 2.1(0.83-5.3) & 0.12 & & \\
\hline \multicolumn{8}{|l|}{ Occupation } \\
\hline House wife & $22(23.2)$ & $17(77.2 \%)$ & $5(22.7 \%)$ & $0.9(0.2-4.5)$ & 0.88 & & \\
\hline Unemployed & $21(22.1)$ & $11(52.4 \%)$ & $10(47.6 \%)$ & $2.7(0.57-13)$ & 0.2 & & \\
\hline Private & $32(38.9)$ & $29(90.6 \%)$ & $8(25 \%)$ & $0.83(0.2-3.8)$ & 0.81 & & \\
\hline Petty Trader & $3(3.2)$ & $3(100 \%)$ & 0 & NA & & & \\
\hline Government & $12(12.6)$ & $9(75 \%)$ & $3(25 \%)$ & 1 & & & \\
\hline \multicolumn{8}{|l|}{ Education } \\
\hline Illiterate & $19(20)$ & $14(73.7 \%)$ & $5(26.3 \%)$ & $0.71(0.13-4.0)$ & 0.7 & & \\
\hline Literate & $8(8.4)$ & $5(62.5 \%)$ & $3(37.5 \%)$ & $1.2(0.16-8.8)$ & 0.86 & & \\
\hline Elementary & $24(25.3)$ & $16(66.7 \%)$ & $8(33.3 \%)$ & $1(0.2-5.1)$ & 1 & & \\
\hline Secondary & $35(36.8)$ & $28(80 \%)$ & $7(20 \%)$ & $0.5(0.1-2.5)$ & 0.4 & & \\
\hline Higher & $9(9.5)$ & $6(66.6 \%)$ & $3(33.3 \%)$ & 1 & & & \\
\hline \multicolumn{8}{|l|}{ Marital status } \\
\hline Single & $21(22.1)$ & $15(\% 71.4)$ & $6(28.5 \%)$ & $1.12(0.23-4.5)$ & 0.87 & & \\
\hline Married & $32(33.7)$ & $26(81.3 \%)$ & $6(18.8 \%)$ & $0.6(0.17-2.5)$ & 0.53 & & \\
\hline Separated & $8(8.4)$ & $6(75 \%)$ & $2(25 \%)$ & $0.93(0.14-6.2)$ & 0.94 & & \\
\hline Divorced & $15(15.8)$ & $8(44.4 \%)$ & $7(46.7 \%)$ & $2.4(0.58-10.3)$ & 0.22 & & \\
\hline Widowed & $19(20)$ & $14(73.7 \%)$ & $5(26.3 \%)$ & 1 & & & \\
\hline \multicolumn{8}{|l|}{ Drinking } \\
\hline Yes & $3(3.2)$ & $2(66.7 \%)$ & $1(33.3 \%)$ & $0.75(0.07-8.6)$ & 0.81 & & \\
\hline No & 92(96.8) & $67(72.8 \%)$ & $25(27.1 \%)$ & 1 & & & \\
\hline \multicolumn{8}{|c|}{ Previous TB history } \\
\hline Yes & $44(46.3)$ & $24(54.5 \%)$ & $20(45.5 \%)$ & $6.2(2.2-17.7)$ & 0.001 & $9.5(3.0-30.3)$ & $<0.001$ \\
\hline No & $51(53.7)$ & $45(88.2 \%)$ & $6(11.8 \%)$ & 1 & & & \\
\hline \multicolumn{8}{|c|}{ Close Contact with TB patient } \\
\hline Yes & $9(9.5)$ & $7(77.8 \%)$ & $2(22.2 \%)$ & $0.74(0.14-3.8)$ & 0.72 & & \\
\hline No & $86(90.5)$ & $62(72.1 \%)$ & $24(27.9 \%)$ & 1 & & & \\
\hline \multicolumn{8}{|l|}{ HIV stage } \\
\hline Stage One & $85(89.5)$ & $62(72.9 \%)$ & $23(27 \%)$ & 1 & & & \\
\hline Stage Two & $3(3.2)$ & $2(66.7 \%)$ & $1(33.3 \%)$ & $1.3(0.12-15.6)$ & 0.81 & & \\
\hline Stage Three & $6(6.3)$ & $4(66.7 \%)$ & $2(33.3 \%)$ & $1.4(0.23-7.9)$ & 0.74 & & \\
\hline Stage Four & $1(1.1)$ & $1(100 \%)$ & 0 & & & & \\
\hline \multicolumn{8}{|l|}{ CD4 count } \\
\hline$\leq 350$ & $32(33.7)$ & 21(65.6\%) & $11(34.4 \%)$ & $1.7(0.66-4.25)$ & 0.3 & & \\
\hline$>350$ & $63(66.3)$ & $48(76.2 \%)$ & $15(23.8 \%)$ & 1 & & & \\
\hline
\end{tabular}

Table 4: Association between risk factors and Radiological finding at St. Paul's Hospital Millennium Medical College.

current and previous TB. Six of these had an X-ray result of highly suggestive for TB while eleven had probable indications and four had non TB lung disease. Fibrosis was seen in ten of the participants, infiltrate in eight and consolidation in six of the persons (Table 3). The finding of abnormal CXR finding from previously TB positive patients was high $(45.5 \%)$, and there was a significant association between final CXR abnormal result and previous TB history $(\mathrm{P}=0.001)$ (Table 4). Patients with previous TB history were more prone to have abnormal chest X-ray than who do not have previous TB. The abnormality rate of X-ray finding among older ages was higher (37.5\%) than younger ages (22.2\%). However, there was no statistical significant association detected between age and CXR findings. Among a total of three alcohol consumer individuals, one patient was with abnormal $\mathrm{X}$ - ray result. However, there was no significant association observed. Bivariant and multivariant logistic regression revealed that only individuals with previous history of TB showed a significant association with abnormal CXR results. Therefore, in this study previous history of TB was an independent predictor for the occurrence abnormality of chest X-ray finding $(\mathrm{AOR}=9.5, \mathrm{CI}=3.0-30.3, \mathrm{P}<0.001)($ Table 4$)[16-18]$.

\section{Discussion}

Ethiopia is a high-burden country for $\mathrm{TB}$, with an estimated incidence of 341 of 100,000 population per year in 2005 [19]. In addition to high TB burden, Ethiopia has been seriously affected by HIV/AIDS, with an estimated 1.5 million HIV-infected persons currently [20]. In the current study, the prevalence of undiagnosed Tuberculosis among asymptomatic HIV- patients was $1.1 \%$, which suggests there is an undiagnosed active TB case. Our finding was similar with an intensified TB case finding study by Sarita Shah where from HIV testing and counseling center in Ethiopia found that $1.14 \%$ 
Citation: Solomon S, Asmare Y, Taddesse B, Negah S, Mamuye Y, et al. (2015) Prevalence of Tuberculosis among HIV Positive Individuals with Asymptomatic Disease States at St. Paul's Hospital Millennium Medical College, Addis Ababa, Ethiopia. J Med Microb Diagn 4: 192. doi:10.4172/21610703.1000192

of all enrolled participants were asymptomatic culture confirmed TB cases [17]. These studies highlight the entity that is subclinical TB. However, this finding was relatively lower than a study in South Africa (3.8\%) undiagnosed cases [21]. This difference might be due to the recruitment criteria, sample size, study

Methodology and other socio and immunological factor. There is also a similar study done on subclinical TB infections on HIV1 population in south Africa which showed a subclinical culture confirmed prevalence of $3.8 \%$ but this might be that South Africa has a very high incidence of $\mathrm{TB}$ with an estimated national incidence rate of 948/100 000 population per year in 2007 [22]. The study in Nepal showed 5.97\%, higher than the current study. The reason that in Nepal, there is a substantial burden of TB disease in HIV infected individuals [23]. Several studies have reported subclinical asymptomatic TB disease as defined by culture-positive $\mathrm{TB}$ in the absence of $\mathrm{TB}$ symptoms. Swaminathan et al. screened HIV-1-infected persons in India and reported a $4 \%$ prevalence of unrecognized TB in HIV-1infected persons with minimal or no symptoms [24]. This study was also higher than our study which might be the reason that they were including minimal subclinical cases. In addition, TB prevalence survey in Zimbabwe found that $41 \%$ of all prevalent cases were subclinical at the time of screening, $82 \%$ of whom had developed symptoms within a few weeks of follow-up; no significant risk factors for prevalent culture-positive disease were found [21]. One theory suggested is that, in a healthcare system with a strong TB diagnosis and treatment programme, severe cases are identified early leaving TB patients with fewer clinical symptoms to be identified through provider-initiated TB screening [21]. In the current study socio-demographic characteristics and related risk factors were not significantly associated with TB positivity rate by smear positive and Xpert assay. This is similar with a study in Nepal, no significant association was found between TB and risk factors like ARV status, common clinical features of tuberculosis and smoking habit [23]. Chest radiography was used to screen for tuberculosis (TB) in asymptomatic persons living with the human immunodeficiency virus. Eight individuals $8.4 \%$ had infiltrate, which is lower than a study by Agizew and his collogues in Gaborone, Botswana with $42 \%$ having infiltrates (36\% upper lobes), 35\% parenchymal fibrosis and 32\% adenopathy [25]. This study in Botswana also showed a $2.3 \%$ abnormal chest $\mathrm{X}$ ray related to $\mathrm{TB}$ of the total enrolled ( $7 \%$ of the abnormal chest $\mathrm{x}$ ray finding). This finding was disagrees with the current study, which showed a $17.9 \%$ abnormal chest $\mathrm{x}$ ray indicating TB. This might be due to the difference in sample size, duration of study period, environmental and immunological status of the participants. In the meantime, presence of a patient with previous TB history was significantly abnormal by chest X-ray finding than a patient without TB history.

\section{Strength and Limitation}

One of the strength of the current study is being a multi-displinary type of research in the setting. The application of Xpert assay among asymptomatic HIV-patient was the first time in the country. The most integral part of the drawback of this study was not using the culture method as a gold standard.

\section{Conclusion and Recommendation}

The present finding of asymptomatic undiagnosed PTB among HIV-patient is $1.1 \%$ (both with AFB and Xpert) in the study area. This showed there is a chance of transmissions of TB to families, friends and close neighborhood before the proper diagnosis and treatment is made. There were $27.4 \%$ abnormal chest X-ray results among asymptomatic patients. Patient with previous TB history was more likely to have abnormal chest X-ray finding than a patient without history of TB. The high abnormal chest X-ray finding rates of chest X-ray result in patients with previous TB history should be taken into consideration. While the symptom screening algorithm missed the $1.1 \%$ TB cases, the AFB technique was able to detect the asymptomatic and missed TB case that was also detected by Xpert MTB/RIF assay. TB control programs must consider TB disease prevalence when estimating the possible performance of any screening algorithm. National TB control program should weigh the risks, benefits and cost of screening all asymptomatic HIV infected individuals for TB by Xpert MTB/RIF assay.

\section{Ethical Review}

Ethical clearance and approval was obtained from St. Paul's Hospital Millennium Medical College Institutional Review Board (IRB) and Ethiopian Public Health Institute Scientific and Ethical Review Committee (SERC). HIV positive individuals were approached by asking their willingness and their permission to participate in the study and signed consent form was obtained from the participants.

\section{Funding}

This research is funded by St. Paul's Hospital Millennium Medical College. The view of the authors are solely their own and do not reflect the views of SPHMMC.

\section{Authors Contributions}

SS conceived, designed, carried out the study and wrote the manuscript,YA advised in proposal development and provided clinical input for the study, SN carried out the X-ray reading. BT confirmed the X-ray reading. YM \& BY carried out the data analysis. ZY \& ET performed the GeneXpert MTB/RIF assay while AK coordinated laboratory work, drafted the proposal and assisted with the write up of the manuscript

\section{Acknowledgement}

We would like to express our deepest gratitude and thanks to the collaborators for their unreserved advice, comment, support and provision of timely and valuable advice for the accomplishment of this work. We express our special gratitude to St. Paul's Hospital Millennium Medical College, for the Provost Dr. Zerihun Abebe and Academic Vice-Provost Dr Lia Tadesse for their vision and thirst of research undertakings and their material and financial support. We gratefully acknowledge the study participants for their willingness to participate in our study. We are also indebted for the nurses, laboratory technologists, radiology technicians, radiologists, data encoders and receptionists of SPHMMC, who screened and recruited participants and devoted their time for facilitating the data collection process. Our heartfelt appreciation goes to Dr. Yibeltal Assefa of EPHI for his kind heart, readiness to do collaborative works with other institutes and for allowing us to use the institute's facility. Credit is also fitting to Dr Eshetu Lemma, for his comments on improving and refining the proposal. Many thank goes out for the staffs of EPHI, for running the samples with Xpert MTB/RIF assay and their dedication for getting the result out on time. Moreover, we pass our regards and thanks to all who supported us in any respect to complete this study.

\section{References}

1. Hopewell $P$, Chaisson $R$ (2000) Tuberculosis and human immunodeficiency virus infection. 2nd ed. New York: Marcel Dekker.

2. WHO (2011) Guidelines for intensified tuberculosis case-finding and isoniazid preventive therapy for people living with HIV in resourceconstrained settings.

3. Smith P, Ross A (1994) Epidemiology of tuberculosis. Washington, D.C: In Bloom BR, ed. Tuberculosis: Pathogenesis, Protection, and Control.

4. van Rie A, Warren R, Richardson M, Victor TC, Gie RP, et al. (1999) Exogenous reinfection as a cause of recurrent tuberculosis after curative treatment. N Eng J Med 341: 1174-1179. 
Citation: Solomon S, Asmare Y, Taddesse B, Negah S, Mamuye Y, et al. (2015) Prevalence of Tuberculosis among HIV Positive Individuals with Asymptomatic Disease States at St. Paul's Hospital Millennium Medical College, Addis Ababa, Ethiopia. J Med Microb Diagn 4: 192. doi: $10.4172 / 21610703.1000192$

5. $\mathrm{MOH}$ (2012) Assessing Progress Towards The Millenium Development Goals. Ethiopia Mdgs Report.

6. Keeler E, Perkins MD, Small P, Hanson C, Reed S, et al. (2006) Reducing the global burden of tuberculosis: the contribution of improved diagnostics. Nature 444 Suppl 1: 49-57.

7. Schluger NW (2003) The diagnosis of tuberculosis: what's old, what's new Semin Respir Infect 18: 241-248.

8. Centers for Disease Control and Prevention (CDC) (2009) Updated guidelines for the use of nucleic acid amplification tests in the diagnosis of tuberculosis. MMWR Morb Mortal Wkly Rep 58: 7-10.

9. Claude L (2010) Tuberculosis: The Essentials. Fourht Edition ed. New York: Informa Healthcare USA, Inc.

10. Gao J, Zheng P, Fu H (2013) Prevalence of TB/HIV co-infection in countries except China: a systematic review and meta-analysis. PLoS One 8: e64915.

11. EHNRI/MOH (2013) National TB/HIV Sentinel Surveillance one year report (2011/12), Addis Ababa, Ethiopia.

12. FMOH (2013) Xpert MTB/RIF Implementation Manual for Ethiopia

13. Lawn SD, Edwards DJ, Kranzer K, Vogt M, Bekker LG, et al. (2009) Urine lipoarabinomannan assay for tuberculosis screening before antiretrovira therapy diagnostic yield and association with immune reconstitution disease. AIDS 23: 1875-1880

14. Achkar JM, Jenny-Avital ER (2011) Incipient and subclinical tuberculosis: defining early disease states in the context of host immune response. J Infect Dis 204 Suppl 4: S1179-1186.

15. Getahun $H$, Harrington $M$, O'Brien R, Nunn $P$ (2007) Diagnosis of smearnegative pulmonary tuberculosis in people with HIV infection or AIDS in resource-constrained settings: informing urgent policy changes. Lancet 369 . 2042-2049.

16. Getahun H, Kittikraisak W, Heilig CM, Corbett EL, Ayles H, et al. (2011) Development of a standardized screening rule for tuberculosis in people living with HIV in resource-constrained settings: individual participant data metaanalysis of observational studies. PLoS Med 8: e1000391.

17. Shah S, Demissie M, Lambert L, Ahmed J, Leulseged S, et al. (2009) Intensified tuberculosis case finding among HIV-Infected persons from a voluntary counseling and testing center in Addis Ababa, Ethiopia. J Acquir Immune Defic Syndr 50: 537-545.

18. Bassett V, Wang B, Chetty S, Giddy J, Losina E, Mazibuko M, et al. (2009) Intensive Tuberculosis Case Finding Among HIV-Infected Persons from a Voluntary Counseling and Testing Center in Addis Ababa, Ethiopia.". Epidemiology and Social Science50: 537-545.

19. Onozaki I (2013) [National surveys of the prevalence of tuberculosis diseaseoverview, progress and lessons learnt]. Kekkaku 88: 777-783.

20. UNAIDS (2006) Report on the Global AIDS Epidemic: A UNAIDS 10th Anniversary Special Edition.

21. Corbett EL, Charalambous S, Moloi VM, Fielding K, Grant AD, et al. (2004) Human immunodeficiency virus and the prevalence of undiagnosed tuberculosis in African gold miners. Am J Respir Crit Care Med 170: 673-679.

22. Oni T, Burke R, Tsekela R, Bangani N, Seldon R, et al. (2011) High prevalence of subclinical tuberculosis in HIV-1-infected persons without advanced immunodeficiency: implications for TB screening. Thorax 66: 669-673.

23. Verma SC, Dhungana GP, Joshi HS, Kunwar HB, Pokhrel AK (2012) Prevalence of pulmonary tuberculosis among HIV infected persons in Pokhara, Nepal. J Nepal Health Res Counc 10: 32-36.

24. Swaminathan S, Paramasivan CN, Kumar SR, Mohan V, Venkatesan P (2004) Unrecognised tuberculosis in HIV-infected patients: sputum culture is a useful tool. Int J Tuberc Lung Dis 8: 896-898.

25. Agizew T, Bachhuber MA, Nyirenda S, Makwaruzi VZ, Tedla Z, et al. (2010) Association of chest radiographic abnormalities with tuberculosis disease in asymptomatic HIV-infected adults. Int J Tuberc Lung Dis 14: 324-331. 